\title{
Efficacy and Reusability of Commercial Adsorbent for Isolation of Proanthocyanidins from Hot Water Extract of Pinus radiata Bark ${ }^{1}$
}

\author{
Sung Phil Mun ${ }^{2 \dagger}$
}

\begin{abstract}
This study was carried out to investigate the feasibility and usefulness of a commercial synthetic adsorbent, Diaion HP 20, for the isolation of proanthocyanidins (PAs) from hot water extract (HWE) prepared from Pinus radiata bark. Most ultraviolet (UV) absorbing materials in HWE were adsorbed onto Diaion HP 20 and easily recovered by simple ethanol (EtOH) washing. More than $50 \%$ of the UV absorbing materials were adsorbed within 20 minutes, and the adsorption equilibrium was reached within $3 \mathrm{~h}$. The recovered materials from Diaion HP 20 were mostly composed of PAs, but some monomeric flavonoids such as taxifolin and unidentified hydrophobic materials were also adsorbed. The impurities such as carbohydrates and inorganic materials contained in HWE were mostly removed by Diaion HP 20 . The adsorption ability of the UV absorbing materials onto Diaion HP 20 was almost the same as the first cycle even after 14 times of repeating cycles of adsorption and desorption. The radical scavenging ability of the recovered materials from the adsorbent was slightly higher than that of the pure PAs prepared by Sephadex LH 20 from the same HWE.
\end{abstract}

Keywords : Pinus radiata bark, Commercial adsorbent, Diaion HP 20, Hot water extract (HWE), Adsorption, Desorption, Proanthocyanidins (PAs), UV absorbing materials

\section{INTRODUCTION}

Recently, we reported that Pinus radiata bark contains not only a great amount of hot water extract (HWE) in comparison with other Pinus species bark but also proanthocyanidins (PAs) (Mun and $\mathrm{Ku}, 2006 ; \mathrm{Ku}$ and Mun, 2007; $\mathrm{Ku}$ et al., 2011). PAs (Fig. 1) are major polyphenols in HWE prepared from $P$. radiata bark and composed of more than $80 \%$ of HWE (Ku et al., 2011; Park et al., 2011). PAs are a potent antioxidant (Priorand $\mathrm{Gu}, 2005 ; \mathrm{Ku}$ and Mun, 2008) and have various physiological effects, such as anti-bacterial agents (Shan et al., 2007), and effective enzyme inhibitors (Stevens et al., 2002; Ku and Mun, 2008). In addition, they also exhibit vasodilatory, anti-allergic, anti-inflammatory (Torras et al., 2005), cardioprotective (Kohama et al., 2004), immune-stimulating, anti-viral, and estrogenic activities (Bagchi et al., 2000).

Currently the majority of $P$. radiata bark discharged from wood related industry has mostly

1 Received February 7, 2014; accepted March 10, 2014

2 Department of Wood Science and Technology, Chonbuk National University, Jeonju, South Korea

† Corresponding author : Sung Phil Mun (e-mail: msp@jbnu.ac.kr) 
<smiles>[R]c1cc([C@H]2Oc3c(c(O)cc(O)c3[C@H]3c4c(O)cc(O)c([C@@H]5c6c(O)cc(O)cc6O[C@H](c6cc([R])c(O)c(O)c6)[C@@H]5O)c4O[C@H](c4cc([R])c(O)c(O)c4)[C@@H]3O)C[C@H]2O)cc(O)c1O</smiles>

$\mathrm{R}=\mathrm{H}$, procyanidin; $\mathrm{R}=\mathrm{OH}$, prodelphinidin

Fig. 1. Chemical structure of proanthocyanidins isolated from Pinus radiata bark.

been used onsite as a boiler fuel to generate steam and power, and a part of the bark is used for mulching and soil treatment in South Korea (Mun et al., 2006). Therefore, the development of economical separation and production methods of PAs, value-added materials, from the bark would be beneficial for further applications and commercialization.

Commercially available and synthetic adsorbent, Diaion HP 20, is a basically styrene polymer and is widely used in a variety of industrial applications, such as adsorption, desalting and decolorization of natural products and small proteins. It is known to be suitable for adsorbing large molecules because of its relatively large pore size and have superior adsorption/desorption ability $\left(\right.$ Diaion $\left.^{\circledR}\right)$.

On the other hand, the main component in HWE is PAs, but some other components such as neutral sugars, inorganic materials, and lowmolecular weight organic compounds, also exist (Mun and Ku, 2006). Therefore, these impurities in HWE should be removed not only for the production of pure PAs; but also for further useful applications, such as health supplements, food additives, and medical supplies. The aim of this study was to explore that PAs can be effectively isolated from HWE using Diaion HP 20. We also investigated the reusability of Diaion HP 20, which can provide useful information for the commercialization of PAs isolation and purification method.

\section{MATERIALS AND METHODS}

\subsection{Materials}

The outer bark of $P$. radiata was provided from Hanyoung Co. Ltd. in Kunsan, South Korea. The bark was dried in a convection oven at $60 \pm 1^{\circ} \mathrm{C}$ for $48 \mathrm{~h}$ and then ground using a Wiley mill installed with a $1 \mathrm{~mm}$ screen. The ground powder was further sieved, and 25 meshpassed powders were used for the preparation of HWE. Diaion HP 20 was purchased from Mitsubishi Chemical (Tokyo, Japan).

\subsection{Preparation of HWE}

Forty grams (based on oven-dried weight) of bark powder and $400 \mathrm{~mL}$ of de-ionized water (DI-water) were taken into a 1-L Erlenmeyer flask (liquor to bark ratio of 10), and then a 1.2-m glass tube as a condenser was attached to the flask. After that the flask was placed on a water bath and allowed to boil for $1 \mathrm{~h}$. The bark slurry after boiling was filtered through a 17G3 glass filter. The residue on the filter was washed with $500 \mathrm{~mL}$ of hot DI-water $\left(>90^{\circ} \mathrm{C}\right)$. The filtrate and washing were all combined together and the total volume of the solution was 
adjusted to 1-L with DI-water. This solution was defined as HWE and subjected to adsorption and desorption study. The residue after the filtration was dried in a convection oven at $105^{\circ} \mathrm{C}$ overnight to calculate HWE yield. The average HWE yield after three runs was $25.7 \%$. The yield of HWE was calculated using the following equation;

$$
\operatorname{HWE}(\%)=[(\mathrm{PB}-\mathrm{R}) / \mathrm{PB}] \times 100
$$

Where, $\mathrm{PB}=$ Oven-dried weight of pine bark powder $(\mathrm{g})$, and $\mathrm{R}=$ Residue weight after hot water extraction $(\mathrm{g})$

\subsection{Adsorption and Desorption of PAs onto Diaion HP 20}

Diaion HP 20 (ca. $11 \mathrm{~mL}$ ), adsorbent, fully soaked and washed with absolute EtOH and DI-water subsequently, and the washed adsorbent was then transferred to a $250 \mathrm{~mL}$ Erlenmeyer flask with $50 \mathrm{~mL}$ of HWE. The adsorption was conducted at room temperature by agitating at $200 \pm 10 \mathrm{rpm}$. To determine the adsorption rate, the agitation was stopped for $1 \mathrm{~min}$ and then $50 \mu \mathrm{L}$ was taken from the supernatant. The solution was transferred to a $20 \mathrm{~mL}$ test tube and $3 \mathrm{~mL}$ of $\mathrm{EtOH}$ was added to the tube. The solution in the tube was mixed well by a vortex mixer for 1 minute and the mixture was subjected to UV analysis (HP 8452 diode array spectrophotometer, HP, USA). UV absorbance was recorded at $280 \mathrm{~nm}$ and the adsorption yield was calculated as follows;

$$
\begin{aligned}
\text { Adsorption }(\%)= & {\left[\left(\mathrm{Abb}_{280 \mathrm{~nm}}-\mathrm{Aba}_{280 \mathrm{~nm}}\right) /\right.} \\
& \left.\mathrm{Abb}_{280 \mathrm{~nm}}\right] \times 100
\end{aligned}
$$

Where, $\mathrm{Abb}_{280 \mathrm{~nm}}=$ Absorbance before adsorption at $280 \mathrm{~nm}$, and $\mathrm{Aba}_{280 \mathrm{~nm}}=$ Absorbance after adsorption at $280 \mathrm{~nm}$.

\subsection{Reusability Test of Diaion HP 20}

To determine the reusebility of Diaion HP 20, adsorption and desorption were repeated 14 times. The washed Diaion HP 20 (ca. $20 \mathrm{~mL}$ ) was transferred to a $250 \mathrm{~mL}$ Erlenmeyer flaskwith $100 \mathrm{~mL}$ of HWE. The adsorption was then performed in the same manner mentioned above, except for $3 \mathrm{~h}$ adsorption time. After $3 \mathrm{~h}$ adsorption, the adsorbent, Diaion HP 20, was recovered by filtration and washed with $500 \mathrm{~mL}$ of DI-water and then treated with $100 \mathrm{~mL}$ of absolute EtOH to desorb the adsorbed materials. The solvent in the filtrate was removed by a rotary evaporator at $43 \pm 2{ }^{\circ} \mathrm{C}$ and then vacuum dried overnight over $\mathrm{P}_{2} \mathrm{O}_{5}$. The desorbed and dried material was weighed for the calculation of adsorption percentage. Adsorption yield was calculated as follows;

Adsorption $(\%)=$ [Weight of desorbed material $(\mathrm{g}) /$ Weight of dried HWE $(\mathrm{g})] \times 100$

\subsection{DPPH Free Radical Scavenging Activity}

Antioxidant activities were measured by DPPH (1,1-diphenyl-2-picrylhydrazyl radical) free radical scavenging activity and determined relative to catechin. Ten milligrams of each sample (desorbed material, catechin, pure PA previously isolated and purified from the same bark using Sephadex LH 20 ( $\mathrm{Ku}$ and Mun, 2007)), and HWE was dissolved in $10 \mathrm{~mL}$ of methanol $(\mathrm{MeOH})$. The sample solution was then diluted 




Fig. 2. Relationship between the adsorption yields of UV absorbing materials and adsorption times at agitation speed of $200 \mathrm{rpm}$.

to $16.7 \mu \mathrm{g} / \mathrm{mL}$ with $\mathrm{MeOH}$. One milliliter of the dilute solution was mixed with $2 \mathrm{~mL}$ of 0.1 $\mathrm{mM}$ DPPH $\mathrm{MeOH}$ solution in a test tube and reacted in a water bath at $25^{\circ} \mathrm{C}$ for $30 \mathrm{~min}$. The absorbance was then measured at $518 \mathrm{~nm}$. Antioxidant activity of each sample was expressed as the scavenged amount of DPPH free radical.

\subsection{Characterization}

FT-IR spectroscopy was performed using a Shimadzu FTIR-8201PC FT-IR spectrophotometer. The IR spectrum using $\mathrm{KBr}$ pellet was recorded in the transmission mode and in the range of $4,000 \sim 400 \mathrm{~cm}^{-1} \cdot{ }^{13} \mathrm{C}$ NMR spectrum was obtained in acetone- $\mathrm{d}_{4} / \mathrm{D}_{2} \mathrm{O}$ using a JEOL JNM-EX 400 spectrometer.

\section{RESULTS AND DISCUSSION}

\subsection{Adsorption Rate}

The pure PAs isolated from $P$. radiata bark has a maximum UV absorbance at $280 \mathrm{~nm}(\mathrm{Ku}$

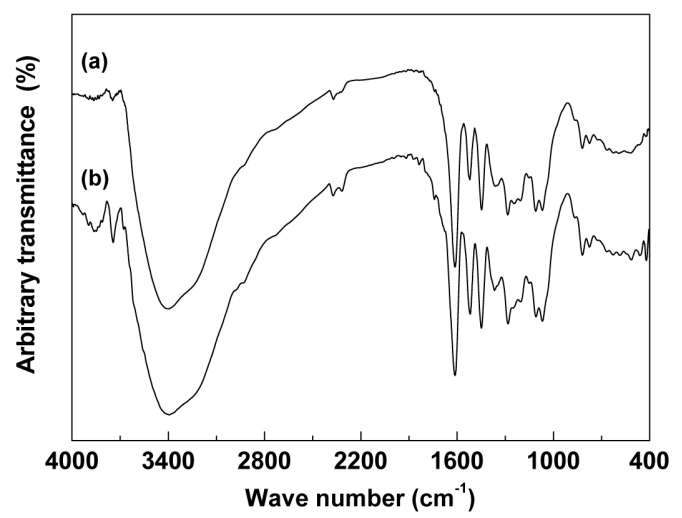

Fig. 3. IR spectra of pure PA prepared from Sephadex LH 20 (a) and the desorbed materials from Diaion HP 20 (b).

and Mun, 2007; Mun et al., 2012). Accordingly, the amounts of the UV absorbed materials onto Diaion HP 20, which are mainly composed of PAs, were measured by recording the absorbance at $280 \mathrm{~nm}$. As shown in Fig. 2, the adsorption was rapidly preceded within $1 \mathrm{~h}$, and more than $50 \%$ of the UV absorbed materials in HWE was adsorbed onto Diaion HP 20 within 20 minutes. The adsorption equilibrium was almost reached in $3 \mathrm{~h}$ and the adsorption yield was about $80 \%$ for the total materials having UV absorbance in HWE. This result indicates that most UV adsorbing materials at $280 \mathrm{~nm}$ in HWE are well adsorbed onto the polystyrene adsorbent, Diaion HP 20. Since this result does not provide information on what sorts of materials are adsorbed, we characterized the adsorbed materials on the adsorbent.

\subsection{Characterization of the Adsorbed Materials}

In the adsorption study using Diaion HP 20 as mentioned above, the materials desorbed after $3 \mathrm{~h}$ adsorption were used for the instrumental 


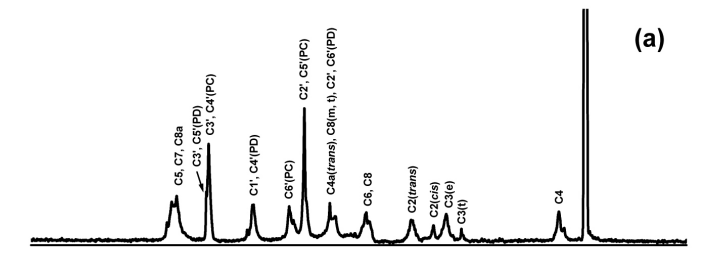

(b)

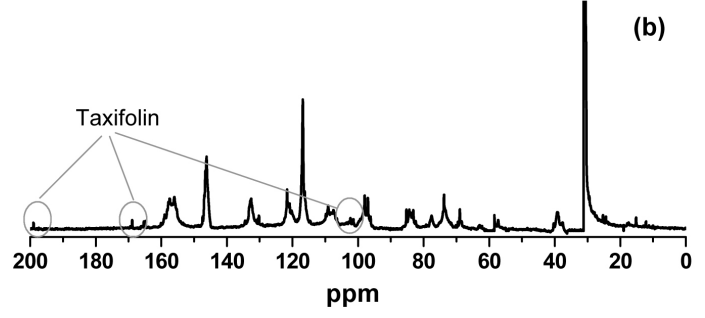

Fig. 4. ${ }^{13} \mathrm{C}$ NMR spectra of pure PA (a) and the desorbed materials onto Diaion HP20 (b).

analysis to characterize their chemical structure. Fig. 3 shows IR spectra of the desorbed materials and the pure PAs isolated by Sephadex LH 20 which was previously reported ( $\mathrm{Ku}$ and Mun, 2007). The IR spectrum of the desorbed materials from Diaion HP 20 was very similar to that of the pure PAs prepared from the same pine bark. Thus, this result indicates that the adsorbed materials are mainly composed of PAs. ${ }^{13} \mathrm{C}$ NMR spectrum as shown in Fig. 4 also supported this result. The same major peaks of the pure PAs on the NMR spectrum also appeared all on the recovered materials from Diaion HP 20. Several small peaks appearing on $198 \mathrm{ppm}$, $164 \sim 168 \mathrm{ppm}, 120 \mathrm{ppm}, 102 \mathrm{ppm}, 85 \mathrm{ppm}$ are all related to taxifolin. We previously reported that taxifolin is one of the main monomeric polyphenols in HWE (Mun and $\mathrm{Ku}, 2006$; $\mathrm{Ku}$ and Mun, 2008). Although Diaion HP 20 is known to be suitable for adsorbing large molecules $\left(\right.$ Diaion $\left.^{\circledR}\right)$, it also adsorbed monomeric polyphenols in HWE. In addition, some hydrophobic materials also appeared in the range from 10 to $25 \mathrm{ppm}$ on the NMR spectrum (Fig. 4b). Most
Table 1. DPPH free radical scavenging activity, based on catechin equivalent antioxidant activity

\begin{tabular}{lc}
\hline \multicolumn{1}{c}{ Samples } & Antioxidant activity \\
\hline \hline Catechin & 1.00 \\
Pure PAs & 0.98 \\
HWE & 0.94 \\
Desorbed materials & 1.11
\end{tabular}

$\mathrm{DPPH}=1,1$-diphenyl-2-picrylhydrazyl radical

carbohydrates as impurities in HWE were removed by using Diaion HP 20. These carbohydrates peaks on the NMR spectrum usually appear in the range from 60 to $80 \mathrm{ppm}$. However, those peaks are almost not existed on the spectrum, although some peaks overlapped with $\mathrm{C}_{2}$ and $\mathrm{C}_{3}$ peaks of PAs. This result indicates that carbohydrates and flavone glycosides in HWE are mostly not adsorbed onto Diaion HP 20. From the FT-IR and ${ }^{13} \mathrm{C}$ NMR spectroscopies of the adsorbed materials, we concluded that Diaion HP 20 is a useful adsorbent for the simple recovery of relatively high purity of PAs from HWE.

In general, PAs has radical scavenging effect because they are a potent antioxidant. Since catechin is a basic unit of PAs, it was used as a positive control in this study. Table 1 shows the result of catechin equivalent DPPH free radical scavenging effect of the adsorbed materials, pure PAs, and HWE. The relative radical scavenging effect of the adsorbed materials was slightly higher than that of HWE and catechin, even higher than the pure PA. Some monomeric polyphenols existing in HWE, such as quercetine, taxifolin, epicatechin, gallocatechin and epigallocatechin, are known to have very strong antioxidant activity (Mun and $\mathrm{Ku}, 2006$; $\mathrm{Ku}$ and Mun, 2008). The DPPH radical scavenging effect of the adsorbed materials may also contrib- 


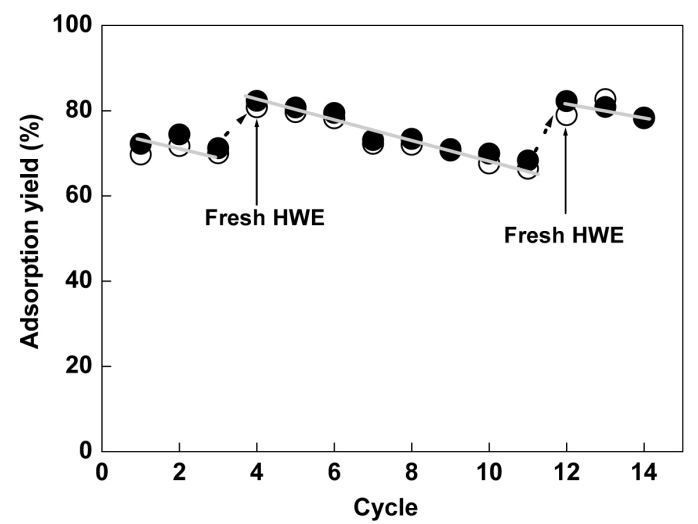

Fig. 5. Reusability of Diaion HP 20 in the isolation of PAs from HWE of $P$. radiata bark.

०: UV method, •: gravimetric analysis.

ute to that these monomeric polyphenols other than taxifolin exist also in them.

\subsection{Reusability of Diaion HP 20}

From the above mentioned results, it was revealed that Diaion HP 20 is an effective adsorbent for the production of relatively pure PAs from HWE. However, for the commercial production of PAs from $P$. radiata bark in the future, the reusability of Diaion HP 20 is very important because the adsorbent price in the process may greatly affect the production cost of PAs. Therefore, we tested the reusability of Diaion HP 20 through 14 times of the adsorption and desorption treatment cycles. Fig. 5 shows the relation between the adsorption yield of the UV absorbing materials and the repeating cycles. By increasing the repeating cycle, the adsorption yields slightly decreased. However, the yields increased again when newly prepared HWE (fresh HWE) was applied. The fresh HWE was in the state of slight cloudy solution but some precipitations start to appear as time passed.
It was thought that the slight decrease in the adsorption yield as the repeating cycle is increased is due to the aggregation of some high-molecular-weight PAs having a poor solubility in water. Even after repeating 14 cycles of adsorption and desorption, Diaion HP 20 still kept all most the same recovery efficiency as the first cycle, and the average adsorption yield was maintained at about $80 \%$. Although slight increase and decrease in the adsorption was observed, we proved that Diaion HP 20 is a very stable and efficient adsorbent for the production of PAs from HWE. In addition, it was revealed that the UV method to determine the adsorption yield would be a useful technique because there were no significant differences in adsorption yield between UV method and gravimetric analysis as shown in Fig. 5.

\section{CONCLUSIONS}

Commercially available synthetic adsorbent, Diaion HP 20, was investigated for the isolation of PAs from HWE prepared from $P$. radiata bark and for its performance through the reusability for the further commercial application. PAs having a strong antioxidant activity in HWE were adsorbed onto Diaion HP 20 within $3 \mathrm{~h}$ and easily recovered by EtOH washing. Although a small amount of monomeric flavonoids and some hydrophobic materials as impurities in the recovered PAs were detected, other impurities that must be removed such as carbohydrates and inorganic materials were mostly removed. A further purification process will be needed for the production of highly pure PAs, but a good performance in its reusability suggested a possible commercial use for the mass production of a moderate pure PAs from 
$P$. radiata bark.

\section{ACKNOWLEDGEMENTS}

This study was carried out with the support of "Forest Science \& Technology Projects (Project No. S211313L010110)" provided by Korea Forest Service. I would like to acknowledge to Franklin Quin, Research Associate II (Mississippi State University, USA), for proof reading of this manuscript.

\section{REFERENCES}

1. Bagchi, D., M. Bagachi, S. J. Stohs, D. K. Das, S. D. Ray, C. A. Ray, S. S. Joshi, and H. G. Pruess. 2000. Free radicals and grape seed proanthocyanidin extract: importance in human health and disease prevention, Toxicol. 148: 187 197 .

2. Diaion ${ }^{\circledR}$, Product Line Brochure, Mitsubishi Chemical Coporation, www.diaion.com.

3. Kohama, T., N. Suzuki, S. Ohno, and M. Inoue. 2004. Analgesic efficacy of French maritime pine bark extract in dysmenorrheal: an open clinical trial, J. Reprod. Med. 49: 828 $>832$.

4. Ku, C. S. and S. P. Mun. 2007. Characterization of proanthocyanidin in hot water extract isolated from Pinus radiata bark, Wood Sci. Technol. 41(3): $235 \sim 247$.

5. Ku, C. S. and S. P. Mun. 2008. Antioxidant properties of monomeric, oligomeric and polymeric fractions in hot water extract from Pinus radiata bark, Wood Sci. Technol. 42(1): 47 60.

6. Ku, C. S. and S. P. Mun. 2008. Stabilization of extracellular matrix by Pinus radiata bark extracts with different molecular weight distribution against enzymatic degradation and radicals, Wood Sci. Technol. 42(5): 427 436.

7. Ku, C. S., S. P. Mun, and J. P. Jang. 2011. Effects of water extraction temperatures on the yield, molecular weight, and antioxidant activity of proanthocyanidins extracted from Pinus radiata bark, Forest Prod. J. 61(4): 321 325.

8. Mun, S. P. and C. S. Ku. 2006. Characterization of low molecular weight polyphenols from pine (Pinus radiata) bark, Food Sci. Biotechnol. 15(3): $424 \sim 430$.

9. Mun, S. P., I. A. Gilmour, and P. J. Jordan. 2006. Effect of organic sulfonic acids as catalysts during phenol liquefaction of Pinus radiata bark, J. Ind. Eng. Chem. 12: 720 726 .

10. Mun, S. P., J. P. Jang, and M. G. Kim. 2012. Effect of Pinus radiata bark polyphenols on phenol liquefaction of cellulose in the presence of $p$-toluene sulfonic acid catalyst, Forest Products J. 62(3): $234 \sim 239$.

11. Park, I. J., S. Y. Cha, M. Kang, Y. S. So, H. G. Go, S. P. Mun, K. S. Ryu, and H. K. Jang. 2011. Effect of Proanthocyanidin-rich extract from Pinus radiata bark on immune responses of specificpathogen-free White Leghorn chickens, Poult. Sci. 90: $977 \sim 982$.

12. Prior, R. L. and L. Gu. 2005. Occurrence and biological significance of proanthocyanidins in the American diet, Phytochem. 66: 2264 2280.

13. Shan, B., Y. Z. Cai, J. D. Brooks, and H. Corke. 2007. Antibacterial properties and major bioactive components of cinnamon stick (Cinnamomum burmannii): activity against food borne pathogenic bacteria, J. Agric. Food Chem. 55: 5484 5490 .

14. Stevens, J. F., C. L. Miranda, K. R. Wolthers, M. Schimerlik, M. L. Deinzer, and D. R. Buhler. 2002. Identification and in vitro biological activities of hop proanthocyanidins inhibition of nNOS activity and scavenging of reactive nitrogen species, J. Agric. Food Chem. 50: 3435 3443.

15. Torras, M. A. C., C. A. Faura, F. Schonlau, and P. Rohdewald. 2005. Antimicrobial activity of Pycnogenol, Phytother. Res. 19: 647 648 . 